\title{
RETRACTED ARTICLE: Comparison of double-bundle anterior cruciate ligament reconstruction with and without autologous conditioned serum application
}

\author{
Nikica Darabos ${ }^{1} \cdot$ Denis Trsek $^{2} \cdot$ Dina Miklic $^{1} \cdot$ Anela Darabos $^{3} \cdot$ Vlasta Brozicevic $^{4}$. \\ Ivan Vlahovic ${ }^{5}$
}

Received: 10 June 2014 / Accepted: 19 November 2014 / Published online: 2 December 2014

(C) European Society of Sports Traumatology, Knee Surgery, Arthroscopy (ESSKA) 2016

This article has been retracted at the request of the corresponding author due to inconsistencies in the described method and incorrect reporting of conflicts of interest.

The online version of this article contains the full text of the retracted article as electronic supplementary material.

Electronic supplementary material The online version of this article (doi:10.1007/s00167-014-3457-8) contains supplementary material, which is available to authorized users.

\footnotetext{
Nikica Darabos

darabos.dr@vz.t-com.hr

1 Department of Sports Traumatology, University Clinic for Traumatology, Clinical Hospital Center Sisters of Charity, 10000 Zagreb, Croatia

2 Special Hospital Akromion, Krapinske Toplice, Croatia

3 The Scientific Unit, General Hospital Varazdin, Varazdin, Croatia

4 Policlinic Terme Selce, Selce, Croatia

5 School of Medicine, University of Zagreb, Salata 3b, Zagreb, Croatia
} 